\title{
Orientation Checklist
}

A P P E N D I X

This is a list of suggested items to be included in an orientation checklist. This is not a competency validation tool.

Date Initiated:

Date Completed:

Preceptor(s):

- Blood Bank location, forms, and procedure to acquire blood and blood products

- Laboratory location, forms, and procedures related to laboratory testing

- Nursery, NICU, postpartum, or mother-baby unit locations

- On-call lists

- Location of telephone numbers for house supervisor, physicians, and other units

- Physical layout of the birthing unit

- Location of labor rooms

- Location of other rooms:

Nursing leader's office

Clean utility (supply) room

Dirty utility room

Medication room

Nurses' changing room

Staff bathroom(s)

Schedule and time clock location

Physicians changing room and sleep (call) rooms

- Other:

Linen storage

Nurses' lounge

Nurses' station

Forms

Whiteboard or computerized patient list

Waiting room information

Visitation rules

Drug dispensing cabinet

Refrigerator(s)

Central monitor

Charts

Prenatal records

Standing orders

Exits

Code cart(s)

Fire extinguishers/evacuation plan

Copyright Springer Publishing Company. All Rights Reserved.
Other:

\section{LABOR ROOM, LDR, OR LDRP}

- Call light/emergency call

- Newborn warmer equipment, medications

- Kick buckets

- Basins

- Sutures

- Yankauer suction

- Warm blankets/blanket warmer

- Bathroom

- Room lights

- Emergency power

- Hand sanitizer (gel)

- Sterile and nonsterile gloves

\section{PATIENT CARE}

- Frontline staff-patient engagement to build trust (scripting)

- Use of the fetoscope and/or Pinard Horn

- Use of the handheld Doppler with simultaneous maternal pulse assessment

- Use of the electronic fetal monitor and fetal monitor Z-fold paper

- Leopold maneuvers

- Application of the ultrasound transducer and the tocotransducer

- Location and use of the ultrasound equipment for a bedside ultrasound by the physician

- Recognition, physiology, and documentation of the following concepts based on institutional expectations and/or requirements:

Accelerations (reactive, nonreactive, spontaneous, and uniform)

Early decelerations

Late decelerations

Variable decelerations (typical and atypical) 
Prolonged decelerations

Spontaneous decelerations

Short-term variability (if spiral electrode is used)

Absent variability

Minimal variability

Average or moderate variability

Marked variability

- Operation of labor/birthing bed

- Operation of bedside table

- Physician remote access to view the electronic fetal/uterine monitor tracings

- Suction:

Wall suction, replacement of canister

Emergency suction equipment

- Oxygen:

Replacing the "Christmas tree"

Location of replacement masks

Non-rebreather mask and reservoir (for delivery of oxygen at $15 \mathrm{~L} /$ minute) versus partial rebreather or simple mask (for delivery of oxygen at $10 \mathrm{~L} /$ minute)

Neopuff: Setup for birth and use

- Precipitous delivery pack (Precip) location and contents

Two Ochsner clamps

One straight Mayo scissors

Four to six towels

One baby blanket

One cord clamp

One straight needle holder

- Location and use of the birthing ball (physio ball)

- Location and use of the peanut ball (based on patient height) and pillows

- Medications refrigerator and storage locations and procedure to obtain (including override protocol)

- IV, amnioinfusion, and blood tubing

- Towels and washcloths

- Warm blankets

- Labels for IV bags and tubing

- Alligator clamps

- Call light, emergency call light, intercom

- Television

- Room lighting

- Computer system

- $\operatorname{Clock}(\mathrm{s})$

- Delivery cart

- Code (emergency) cart

- Anesthesia cart

- Sharps containers (location and procedure to replace them)

\section{ANTEPARTAL SKILLS}

A detailed skills checklist for each procedure and observation by an expert obstetric clinician should be used to confirm competency.
- Nonstress test

- Contraction stress test (oxytocin challenge test)

- Triage assessment and prioritization

- Patient charges

- Documentation

\section{LABOR AND DELIVERY SKILLS AND POLICIES/PROCEDURE/PROTOCOLS}

- Sterile vaginal examination (including assessment of caput, molding, and position)

- Insertion of an IV catheter

- Use of an IV infusion pump

- Anesthesia pump (rules for use)

- Amnioinfusion

- Administration of IV insulin (insulin drip)

- Administration of magnesium sulfate

- Cesarean section

- Fetal monitoring (auscultation vs. continuous)

- Spiral electrode application (if it is an approved nursing skill)

- IUPC insertion (if it is an approved nursing skill)

- Setup for vaginal delivery

- Setup of newborn warmer if there is meconium and if there is no meconium

\section{IMMEDIATE CARE OF THE NEWBORN AND/ OR MULTIPLES (TWINS, TRIPLETS, ETC.):}

- Airway and suctioning

- Temperature control

- Oxygen administration APRN

- Physical examination

- Apgar scores

- Documentation (including late entries)

- Assisting with a vacuum extraction and a double setup

- Assisting with a forceps delivery and a double setup

- Patient charges

- Documentation of operative vaginal delivery (including late entries)

- Vaginal delivery

\section{RECOVERY SKILLS}

- Fundal and lochia checks

- Vital sign checks

- Perineal care

- Removal of the epidural tubing (following competency validation)

- Report to postpartum nurse 


\section{OPERATING ROOM SKILLS}

- Preparation of surgical site

- Preparation for cesarean section:

- Identification band on and information verified as accurate

- Allergies noted

- Informed consent process verified and consent signed

- Preoperative orders reviewed

- Scrub nurse or scrub tech notified

- Anesthesia personnel notified

- Neonatal care personnel notified

- Preoperative medications administered

- Foley catheter inserted

- Patient transfer to operating room (OR) table and legs secured

- Grounding pad applied to thigh and documented

- Cautery unit set to surgeon's preferences and documented

- Fetal heart rate assessed and documented (and maternal heart rate differentiated from acquired rate) prior to abdominal prep

- Surgeons' gowns tied

- Time-out completed

- Documents using form and/or software in the OR

- Opens packs using aseptic technique

- Adds instruments using aseptic technique

- Performs cricoid pressure when requested

- Properly labels and prepares specimens

- Addition of sterile supplies to back table

- Pouring sterile solutions into splash basins

- Sponge, needle, and instrument counts

- Neonatal warmer operable and equipped

- Newborn paperwork and identification bands properly completed

- Assists with moving patient from table to bed

- Applies warm blankets to patient

- Safely transports patient to recovery room

- Gives SBAR report to recovery room nurse

- Observed aseptic technique at all times

- Limited conversation

- Protected patient's modesty

- Never left patient unattended

- Other:

\section{LABOR ROOM SUPPLIES}

- Examination gloves (application and removal)

- Sterile lubricant location and use

- Device for rupturing the membranes

- Linen protector location

- Scale (to weigh bloody pads or linen protector or towels)

- Peri-pads
- Sterile specimen containers

- Hair clipper (for the abdomen prior to cesarean section)

- Bedpan

- Birthing bed (operation and setup for delivery)

- Warming devices

- Culturette swabs

- Endotracheal tubes (adult and neonatal)

- Epidural cart/supplies

- Spirometer, adult

- Staple removal set

- Spinal needles: 22 gauge, 27 gauge

- Spiral electrode and cable

- Compression stockings and/or sequential compression devices (SCDs)

- Intrauterine pressure catheter and cable

- IV solutions

- IV start kit or supplies

- IV catheters

- IV tubing

- IV pumps

- Amnioinfusion tubing

- IV extension tubing

- Microdrip tubing

- Blood tubing

- Additional IV pumps, IV poles (storage location)

- Foley catheter

- Amniocentesis tray

- Adult oxygen mask

- Oxygen extension tubing

- Graduated cylinder

- Commode container hat

- 24-hour urine collection container

- Clean-catch urine supplies

- Red Robinson (straight) catheter

- Perineal pads/linen protectors/Chux

- Spiral electrode and cable

- Stopcocks

- Transducer belts

- Shampoo

- Hand lotion

- Facial tissues

- Disposable panties

- Peri bottle (for cleansing after delivery)

- Red bags

- Other:

\section{OTHER SUPPLIES}

- Physician preferences cards

- Pudendal tray

- Skin-prep tray

- Betadine scrub/solution

- OB pack

- Gown pack 
- Gloves

- Sutures

- Lap sponges

- Gauze (radio-opaque) $4 \times 4$ s

- Mayo stand cover

- Drape sheet

- Disposable speculum

- $1,500 \mathrm{~mL}$ sterile water

- $1,000 \mathrm{~mL}$ sterile water

- 1,500 mL normal saline

- Disposable "ice" pack

- Suction canister

- Suction tubing

- Cautery unit and pad

- Vacuum extractor and additional cups (bell and posterior [M/Mushroom] cups)

- Forceps

- Cord blood gas collection equipment and technique

- Warm blankets

- Specimen containers

- Adhesion prevention supplies

- Positioning wedge

- Other:

\section{INSTRUMENTS THAT MAY BE USED DURING} AND AFTER A VAGINAL DELIVERY

- Two ring forceps

- Two towel clips

- Two hemostats

- Six Allis clamps

- Two Ochsner clamps

- One needle holder

- One tissue forceps with teeth

- One curved Mayo scissor

- One straight Mayo scissor

- One instrument stringer

- Six towels

- One small prep cup

- One medicine cup

- Also: sterile sutures, disposable scalpel and blade, needles

- Mand syringes

INSTRUMENTS THAT MAY BE USED TO REPAIR A LACERATION

- Four ring forceps

- Five Allis clamps

- One uterine dressing forceps

- Two Heaney retractors

- One large curved Mayo scissors

- One short straight scissors

- One large needle holder
- One short needle holder

- One Heaney needle holder

- One large tissue forceps with teeth

- One short tissue forceps with teeth

- One vaginal speculum

- One instrument stringer

- Six towels

\section{INSTRUMENTS THAT MAY BE USED FOR A DILATATION AND CURETTAGE (D\&C)}

- Four ring forceps

- One Heaney scissor

- One straight Mayo scissor

- One needle holder

- One vaginal speculum

- One weighted vaginal speculum

- One Heaney retractors

- One large curette

- One instrument stringer

- Six towels

\section{INSTRUMENTS THAT MAY BE USED DURING A CESAREAN SECTION}

- Eight curved hemostats

- Four Allis clamps

- Four Penningtons

- Two Babcock clamps

- One curved Kocher clamp

- Four Ochsner clamps

- Two Heaneys

- Two ring forceps

- Two needle holders

- Six tissue forceps: Two with teeth, one smooth, two Adson-Brown, and one Russian

- Five scissors: One straight Mayo, one short, curved Mayo, one long Mayo, one bandage, and one Metzenbaum

- One \#3 knife handle with \#21 blade

- One \#4 knife handle with \#10 blade

- Three retractors: One bladder blade, one large Richardson-Eastman, and one small RichardsonEastman

- One instrument rack with two parts

\section{INSTRUMENTS FOR A HYSTERECTOMY}

- Four ring forceps

- Four Kocher clamps

- Four Rochester-Peons

- Four straight Heaney-Ballentines

- Four curved Heaney-Ballentines 
- Four long Allis clamps

- Two Collier hemostats

- Four tissue forceps: One long with teeth, one long smooth, one long Russian, and one Heaney

- Four scissors: One Jorgensen, one long straight Mayo, one long, curved Mayo, and one Metzenbaum

- Three needle holders: Two straight and one Heaney

- One long \#3 knife handle with \#21 blade

- Six retractors: Two Deavers and two Malleables

- One Balfour blade

- One Balfour retractor with two or three screws

- One instrument rack with two parts

\section{INSTRUMENTS FOR A TUBAL LIGATION}

- Six curved hemostats

- Four Allis clamps

- Two Babcocks

- Two needle holders

- Three scissors: One short, curved; one short, straight; and one Metzenbaum

- Three forceps: One with teeth, one smooth, and one Russian

- Four retractors: Two small Richardson-Eastman and two Army-Navy

- Two \#4 knife handles with \#10 and \#15 blades

\section{INSTRUMENTS AND SUPPLIES FOR A} CERVICAL CERCLAGE

- Two ring forceps

- One large Mayo scissors

- One large, smooth tissue forceps

- One vaginal retractor

- One Heaney needle holder

- One straight needle holder

- Six towels

\section{NEWBORN SUPPLIES AND EQUIPMENT}

- Blankets, thermal caps

- Bulb syringe

- Stethoscope

- Suction catheters

- Suction tubing
- Suction canister(s)

- Endotracheal tubes, stylets, laryngoscope

- Oxygen masks: preemie, term newborns

- Oxygen blender

- Oxygen extension tubing

- Neonatal resuscitation bag

- Newborn pulse oximeter

- Predelivery warmer setup

- Other

\section{NEWBORN CARE}

- Setup for meconium

- Setup for intubation

- Assisting with neonatal resuscitation

- Neonatal Resuscitation Program or similar program of study completion

- Neopuff setup and use

- Participation in neonatal resuscitation

- Other

\section{OPERATING ROOM}

- Review of aseptic technique

- Preparation of neonatal warmer

- Count before procedure

- Count during procedure

- Count upon closure of the uterus

- Documentation in the OR

- Other

\section{RECOVERY ROOM (PACU)}

- Operation of bed

- Operation of patient warming blanket

- Cardiac monitor paper:

Set speed

Changing the paper

Post-monitor strip in chart

- Vital signs equipment

- Medications: Location and procedure to obtain

- Documentation

- Charges for patient care and supplies

- Sign-out procedure(s)

- Other 